\title{
Perioperative Therapy for Borderline Resectable Pancreatic Cancer: What and When?
}

\author{
Michael P. Kim, MD and Matthew H. G. Katz, MD \\ Department of Surgical Oncology, The University of Texas MD Anderson Cancer Center, Houston, TX
}

Over recent years, multidisciplinary teams have increasingly treated patients with borderline resectable pancreatic cancer (BRPC) with chemotherapy and/or radiation before anticipated pancreatectomy. The goal of preoperative treatment remains largely twofold: (1) to downsize the size of tumor and its involvement with adjacent anatomic structures, thereby maximizing the likelihood of a microscopically complete (R0) resection, and (2) to identify patients with pancreatic tumors exhibiting a "locally dominant phenotype"-tumors at low risk for rapid progression and recurrence-for whom pancreatectomy may be most beneficial. Although this strategy is recommended in national treatment guidelines, no clinical trial has prospectively randomized patients to either preoperative therapy or surgery de novo, and data supporting this approach have largely been derived from relatively small, retrospective series. ${ }^{1-3}$ The optimal treatment algorithm for patients with BRPC therefore remains a matter of significant debate. ${ }^{4}$

In this issue of Annals of Surgical Oncology, Nagakawa et al. communicate the results of a propensity score-matched comparison of patients with BRPC treated primarily with either surgery or preoperative therapy. ${ }^{5}$ Their study also compared the outcomes of patients who received preoperative chemotherapy to those who received preoperative chemoradiation. In summary, they found that patients with BRPC who received preoperative therapy before anticipated resection underwent pancreatectomy less frequently than those brought to the operating room de novo. However, pretreated patients underwent R0 resection

\footnotetext{
(C) Society of Surgical Oncology 2019
}

First Received: 31 December 2018; Published Online: 24 January 2019

M. H. G. Katz, MD

e-mail: mhgkatz@mdanderson.org more frequently and as a group lived significantly longer (median survival 25.7 vs. 19 months, $p=0.015$ ). Among the subgroup of patients treated with preoperative therapy, the median overall survival duration of patients who received chemoradiation was similar to that of those who received chemotherapy (22.5 vs. 29.2 months, $p=0.13$ ). Although patients who received chemoradiation underwent pancreatectomy less frequently than those who received chemotherapy, they had a lower rate of local recurrence following resection.

Key elements of the study by Nagakawa et al. deserve recognition. This analysis of treatment algorithms for localized pancreatic cancer is one of the largest and most comprehensive of its kind. This study also reports outcomes of patients treated only at centers that specialize in pancreatic surgery, ensuring a relatively standardized template of multidisciplinary treatment and surgical experience. And, anatomic staging of localized pancreatic cancer requires specialized imaging and the identification of specific radiographic features, the criteria for which tend to be incompletely aligned, even among high-volume pancreatic treatment centers. ${ }^{3}$ In the current study, imaging was reviewed to establish disease stage based on specific, common criteria, ensuring an anatomically homogenous study cohort.

Other aspects of this study, however, deserve scrutiny. The limitations of propensity-score matched analyses, for example, have been well-documented and are particularly dependent on criteria that investigators designate as important. ${ }^{6}$ In this analysis of 884 patients, 56\% (297/530) of pretreated BRPC patients and 84\% (297/354) of patients who underwent surgery primarily were propensity scorematched and analyzed in an attempt to minimize bias. Unexcluded or unmeasured covariates, lingering selection bias, study dropout, and other factors may have resulted in alterations to the study populations included in the final, matched analysis. Nevertheless, the results communicated 
by Nagakawa et al. largely echo previously published reports that suggest—at least as a group — that patients with pancreatic tumors that appear to have a significant radiographic interface with the central mesenteric vasculature benefit from treatment with systemic chemotherapy prior to anticipated pancreatectomy. ${ }^{7,8}$

Moving forward, an obvious question remains. How do we take the results of this study, in which patients were treated between 2011 and 2013 in Japan, and use them to inform the treatment of patients treated worldwide in 2019? Outside Japan, FOLFIRINOX and Gemcitabine plus Abraxane are the favored systemic chemotherapy regimens. Most patients included in this study were treated with the cytotoxic agent $\mathrm{S}-1$, a drug that is unavailable in many countries, including the United States. Furthermore, in this study, most patients who were treated with radiation received a conventional dose of 50-60 Gy, which may take as long as 6 weeks to deliver. General interest among radiation oncologists has now shifted toward rapid-fractionated regimens that allow delivery of relatively high doses of radiation in a duration as short as a few days, possibly without an adverse effect on outcomes. ${ }^{9}$ To the extent that systemic chemotherapy appears to prolong survival and radiation appears to improve local control, shortcourse treatment with radiation therapy following systemic chemotherapy is an appealing strategy that may allow patients the theoretical benefits of both treatment regimens.

Recently, the Gastrointestinal PRODIGE 24/Canadian Cancer Trials Group PA.6 study has renewed doubt about the optimal timing of perioperative therapies. The PRODIGE authors found that postoperative treatment with FOLFIRINOX resulted in a median survival of 54.4 months versus 35 months with single agent gemcitabine. ${ }^{10}$ Given this overall survival duration-longer than any previous report in the prospective treatment of patients with pancreatic cancer-it is evident that multidisciplinary groups should focus on determining which drugs should be delivered, to whom and when, rather than simply making broad statements regarding optimal treatment sequencing algorithms in pancreatic cancer patients as a whole. In coming years, the prospective identification of molecular features that predict sensitivity or resistance to treatment with FOLFIRINOX, gemcitabine, and abraxane and other agents will likely empower physicians to individualize effectively therapies and sequencing algorithms. ${ }^{11,12}$ The influx of novel organoid model systems and sophisticated sequencing technology into the field will likely enable the analysis of genetic and molecular data within the context of individual tumors, providing glimpses into the biology of pancreatic cancer at an unparalleled resolution. Emerging challenges therefore will be to understand and match tumor biology with patient therapy at the expense of what will most certainly be a very steep learning curve.
Certainly, movement toward the goal of all clinical oncologists- to understand and target vulnerable biologic features specific to each patient's tumor and physiologic status-is sorely needed in pancreatic cancer to improve survival outcomes that have remained poorly stable for decades. We look forward to the discovery and strategic implementation of more efficacious therapies in parallel with surgical extirpation and hope that conversations related to the care of patients with this difficult-to-treat stage of pancreatic cancer grow by leaps and bounds.

\section{REFERENCES}

1. Khorana AA, Mangu PB, Berlin J, et al. Potentially curable pancreatic cancer: American Society of Clinical Oncology Clinical Practice Guideline. J Clin Oncol. 2016;34:2541-56.

2. Tempero MA, Malafa MP, Al-Hawary M, et al. Pancreatic adenocarcinoma, version 2.2017, NCCN clinical practice guidelines in oncology. J Natl Compr Cancer Netw. 2017;15:1028-61.

3. Katz MH, Marsh R, Herman JM, et al. Borderline resectable pancreatic cancer: need for standardization and methods for optimal clinical trial design. Ann Surg Oncol. 2013;20:2787-95.

4. Bockhorn M, Uzunoglu FG, Adham M, et al. Borderline resectable pancreatic cancer: a consensus statement by the International Study Group of Pancreatic Surgery (ISGPS). Surgery. 2014;155:977-88.

5. Nagakawa Y, Sahara Y, Hosokawa Y, et al. Clinical impact of neoadjuvant chemotherapy and chemoradiotherapy in borderline resectable pancreatic cancer: analysis of 884 patients at facilities specializing in pancreatic surgery. Ann Surg Oncol. 2019. http s://doi.org/10.1245/s10434-018-07131-8.

6. Brooks JM, Ohsfeldt RL. Squeezing the balloon: propensity scores and unmeasured covariate balance. Health Serv Res. 2013;48:1487-507.

7. Cloyd JM, Crane CH, Koay EJ, et al. Impact of hypofractionated and standard fractionated chemoradiation before pancreatoduodenectomy for pancreatic ductal adenocarcinoma. Cancer. 2016;122:2671-9.

8. Katz MH, Fleming JB, Bhosale P, et al. Response of borderline resectable pancreatic cancer to neoadjuvant therapy is not reflected by radiographic indicators. Cancer. 2012;118:5749-56.

9. Katz MHG, Ou FS, Herman JM, et al. Alliance for clinical trials in oncology (ALLIANCE) trial A021501: preoperative extended chemotherapy vs. chemotherapy plus hypofractionated radiation therapy for borderline resectable adenocarcinoma of the head of the pancreas. BMC Cancer. 2017;17:505.

10. Conroy T, Hammel P, Hebbar M, et al. FOLFIRINOX or gemcitabine as adjuvant therapy for pancreatic cancer. $N$ Engl $\mathrm{J}$ Med. 2018;379:2395-406.

11. Tiriac H, Belleau P, Engle DD, et al. Organoid profiling identifies common responders to chemotherapy in pancreatic cancer. Cancer Discov. 2018;8:1112-29.

12. Aguirre AJ, Nowak JA, Camarda ND, et al. Real-time genomic characterization of advanced pancreatic cancer to enable precision medicine. Cancer Discov. 2018;8:1096-111.

Publisher's Note Springer Nature remains neutral with regard to jurisdictional claims in published maps and institutional affiliations. 